\title{
AVANCES EN EL CONOCIMIENTO ECOLOGICO DE GRACILARIA VERRUCOSA EN EL AREA DE CONCEPCION, CHILE
}

\author{
VICTOR DELLAROSSA, HÉCTOR ROMO Y KRISLER ALVEAL \\ Institu to de Biología, Universidada de Concepcion, Chile
}

\section{Introducción}

La explotación industrial de varias especies de algas marinas, especialmente agarófitas, determinó que diversas instituciones chilenas (Instituto de Fomento Pesquero, Servicio Agrícola y Ganadero; Universidades) iniciaran estudios de poblaciones sublitorales útiles al hombre.

Desde el punto de vista económico, las especies del género Gracilária son las más importantes, siendo la región del Bío Bío una de las áreas productoras más significativas, por la magnitud del recurso que en ella existe y por la diversidad de ambientes en que vive en forma natural.

Hasta la fecha no se han estlabecido normas apropiadas para su extracción; pero se está solicitando bases científicas para lograr una explotación más racional del recurso. En esta búsqueda de información, la Universidad de Concepción ha logrado, en el tiempo, aportar antecedentes referentes a:

- Características de algunos parámetros físico-químicos en los ambientes de crecimiento de Gracilaria.

- Variaciones en el tiempo y en el espacio de la densidad, standing crop y tamaño de las frondas.

- Estimaciones sobre la productividad de estas poblaciones.

- Comportamiento de los estados reproductivos en el ciclo de vida.

- Interacciones biológicas entre Gracilaria y otras especies presentes en las poblaciones.

El área cubierta por estos estudios comprende las Bahías Coliumo, Concepcion y San Vicente; observaciones complementarias han sido efectuadas en el Golfo de Árauco e Isla Santa María $\left(36^{\circ} 32^{\prime} \mathrm{S}-37^{\circ} 22^{\prime} \mathrm{S}\right)$

\section{Aspectos Reproductivos}

La Tabla I resume algunas características reproductivas de las poblaciones de $G$. verrucosa en la región del Bío Bío. En las praderas submareales cistocárpicas y tetrespóricas de Bahía San Vicente (Dellarossa, 1974) y Bahía Concepción (Romo \& Alveal, $1979)$, no se ha detectado indicios de reproducción por médio de esporas, por lo que se infiere que en este tipo de ambientes las poblaciones se mantienen por fragmentación y reproducción nadas por la uniformidad que presentaba la vegetación al momento de iniciar el trabajo. Se ubicó dos stands en Bahía San Vicente, para observar las diferencias locales encontradas previas al estudio, entre centro y borde de la población y uno en Bahía Coliumo para comparar poblaciones geográficamente distantes.

Là extensión de cada stand fue de aproximadamente $500 \mathrm{~m}^{2}$ la de las parcelas de $12 \mathrm{~m}^{2}$. Mensualmente se extrajo por buceo autónomo todo el material de una parcela, superponiendo en ella un cuadrado metálico de las mismas dimensiones y dividido en sectores de $1 \mathrm{~m}^{2}$. La extración del alga se realizó por corte mecánico de ella a un par de centímetros sobre el sustrato e individualizando los haces de frondas para estimar su densided. Al mes siguiente, se procedió de igual forma; pero la nueva parcela se ubicó al otro lado de la boya principal y en forma opuesta a la del mes anterior, para evitar la influencia del efecto de corte (Fig. 1).

Para la población ubicada en Bahía Concepción se fijó en stand en forma similar, obteniéndose muestras al azar con el objeto de determinar el stock de algas a través de las variaciones de standing crop y el comportamiento reproductivo de las plantas.

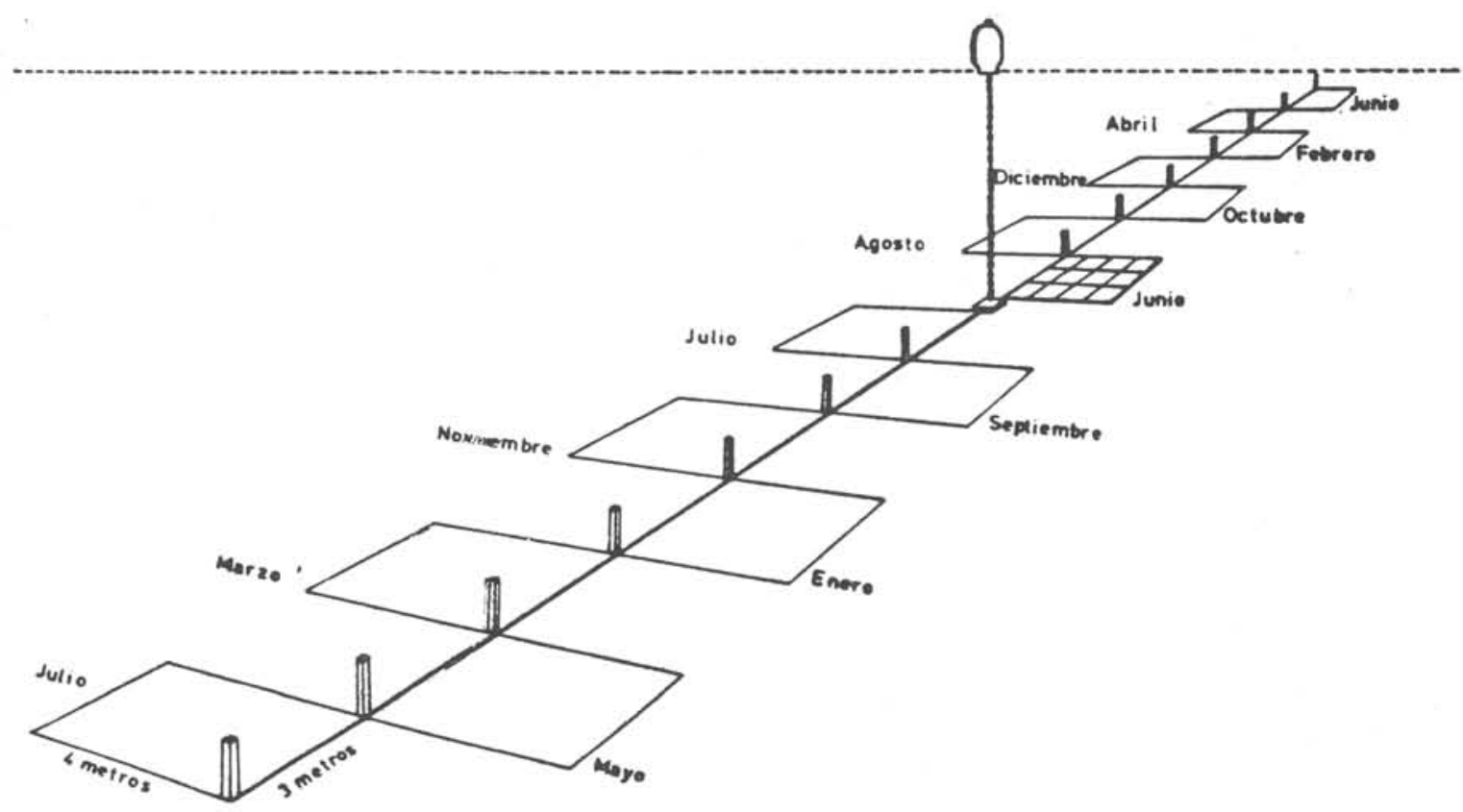

Fig. 1 - Forma, tamaño y distribución de las parcelas permanentes en los stands estudiados.

\section{Materiales y Metodos}

Cubriendo el área de vegetación se ubicó estaciones hidrográficas en forma permanente, en las que se realizó un muestreo quincenal de salinidad, temperatura y oxígeno, considerando los niveles de superficie y fondo.

El estudio de la población se realizó por medio de stands demarcados por una línea base que se fiió con tres boyas y respecto a la cual se situó parcelas permanentes. Estas áreas fueron seleccio- vegetativa, aun cuando en cultivos de laboratorio sus tetrásporas y carpósporas germinan y se cumple la alternancia de generaciones.

Es posible que en algunos ambientes, factores de sustrato y temperatura sean los responsables de la no germinación de esporas en el medio natural. En primer lugar, la inestabilidad del sustrato arenoso umpediría la fijación de esporas y, en segundo lugar bajas temperaturas del mar no seríam apropiadas para su germinación, Las temperaturas superficiales registradas para Bahía Concepción varian entre 10.3 y $16.8^{\circ} \mathrm{C}$, con un promedio de $12.9^{\circ} \mathrm{C}$ 
TABLA I

ALGUNAS CARACTERISTICAS REPRODUCTIVAS DE POBLACIONES DE Gracilaria EN BAHIA CONCEPCION

\begin{tabular}{|c|c|c|c|c|}
\hline \multirow[b]{2}{*}{$\begin{array}{l}\text { TIPO DE } \\
\text { POBLACION }\end{array}$} & \multirow[b]{2}{*}{ - FASES } & \multicolumn{2}{|c|}{ HABITAT } & \multirow[b]{2}{*}{ REPRODUCCION } \\
\hline & & $\begin{array}{l}\text { UBICACION } \\
\text { COSTERA }\end{array}$ & SUSTRATO & \\
\hline \multirow[t]{2}{*}{ MONOFASICA } & tetrasporofita & submareal & $\begin{array}{l}\text { arenoso } \\
\text { fangoso }\end{array}$ & vegetativa \\
\hline & $\begin{array}{l}\text { carposporofita } \\
\text { en planta } ?\end{array}$ & submareal & $\begin{array}{l}\text { arenoso } \\
\text { fangoso }\end{array}$ & vegetativa \\
\hline TRIFASICA & $\begin{array}{l}\text { tetrasporofita } \\
\text { gametofita } \subsetneq \delta \\
\text { carposporofita } \\
\text { en } \varsubsetneqq\end{array}$ & intermareal & rocoso & $\begin{array}{l}\text { sexuada y } \\
\text { por esporas }\end{array}$ \\
\hline
\end{tabular}

(Ahumada \& Chuecas, 1979l. Al respecto, Ogata et al.(1972) señalan que en condiciones experimentales, temperaturas por sobre los $20^{\circ} \mathrm{C}$ son favorables para el desarrollo de las esporas de estā especie; Tsekos \& Karataglis (1974) demostraron que $21^{\circ} \mathrm{C}$ es la temperatura óptima para la germinación de carpósporas de $G$. confervoides (L.) Greville siendo esta especie un sinónimo de G. vernucosa (Hudson) Papenfuss. Kim \& Candia (1978) indican que temperaturas hajo $10515^{\circ} \mathrm{C}$ impiden la maduración de los esporangios de la misma especie.

Las condiciones de sustrato y temperatura apropiadas se presentan en las áreas intermareales rocosas de Bahía Concepción, cumpliéndose aquí la alternancia de generaciones $\mathrm{y}$, por lo tanto, los elementos reproductores son viables.

Observaciones Ecologicas sobre las Poblaciones del Genero Gracilaria.

Las praderas de incidencia comercial más importantes que existen en el litoral del país se encuentran desde Coquimbo a Chiloé; algunas de ellas se presentan en las costas de algunas islas, otras en áreas con permanente afluencia de agua dulce y en la zona sur, de preferencia, en el lecho mismo de algunos ríos en zonas influenciadas por la acción de las mareas. Un rasgo común a todas ellas es su presencia sobre sustrato de arena o fango, ocasionalmente sobre rocas y en número reducido de ejemplares.

Las poblaciones son monoespecíficas (Kim, 1970) y las frondas irregularmente ramificadas; bajo el sustrato se encuentran frondas más viejas que se caracterizan por su mayor grosor y color más oscuro, son éstas las que por actividad vegetativa originan las numerosas ramificaciones que emergem del sustrato, formando haces perfectamente diferenciables como para estimar su densidad.

El rápido crecimiento de las frondas que hace posible un continuo aumento del stock de algas en los meses de crecimiento, su capacidad para vivir en sustrato de arena y fango, la madurez de los órganos reproductores en un breve lapso, sus características de especie euritérmica y eurihalina, son adaptaciones para sobrevivir en el habitat en que se les encuentra.

En $G$ verrucosa se observó que las frondas que emergen del sustrato por actividad vegetativa, poseen un ciclo anual definido; en cambio, tas que están inmersas en él, perduran a la estación desfavorable y comienzan el rebrote de ramificaciones en la época de crecimiento siguiente. Esta información impide considerar el alga como una especie anual.

El Sistema de Katada de Formas de Vida (Katada,1963) al inchuir no sólo el ciclo vital, sino también las partes que perduran a la estación desfavorable, ubica a $G$. verrucosa como una especie perenne, cuyas frondas de sustentación sobreviven en el tiempo; el mismo autor agrega que como resultado de sus experimentos de propagación, la parte perenne se renovaría cada tres anos, observación sobre la que no se posee antecedentes para las poblaciones de la zona.

El establecer la forma de vida de esta especie, permite comprender las características encontradas para las poblaciones estudiadas, sus fluctuaciones en el tiempo y en el espacio y su productividad.

\section{Caracteristicas Abioticas}

Estudios que consideran aspectos ecológicos de este género, indican que se presenta en ambientes donde la temperatura y salinidad'tienen una gran fluctuación en el año (Causey et al., 1944; Stokke, 1957; Ohmi, 1958; Jones, 1959).

Los resultados obtenidos confirmaron un rango amplio de los valores de salinidad y temperatura, pero sólo en las estaciones de superficie; en las de fondo, que caracterizan el ambiente real de estas pohlaciones. se encontró una salinidad típica de un habitat marino, entre 30.7 y $34.5 \%$ en Bahía San Vicente y entre 31.2 y $34.6 \%$ en Bahía Coliumo.

La temperatura puede considerarse como relativamente estable entre 11.0 y $13.0^{\circ} \mathrm{C}$ en Bahía San Vicente y entre 10.8 y $12.8^{\circ} \mathrm{C}$ en Bahía Coliumo.

Los conceptos de dinámica estuarina explican la situación encontrada respecto a temperatura y salinidad. Según Cameron \&. Pritchard (1963) en este tipo de ambiente la magnitud de los cambios en la concentración de sales no es proporcional a la intensidad del flujo de agua dulce, en consecuencia, se encontrará siempre una estratificación vertical de la salinidad.

Respecto a las poblaciones que se presentan en el lecho de ríos, en áreas próximas a su desembocadura y la de las costas de algunas islas cercanas al continente, estos parámetros no han sido estudiados.

\section{Características Bioticas}

Primó el criterio de utilizar las frondas emergentes para conocer las variaciones en el tiempo de la población, puesto que técnicamente resultó imposible cuantificar las que permanecen en él.

Se asumió que la producción neta durante el período de crecimiento podía ser determinada por los cambios en standing crop al incrementar el stock de algas de un mínimo a un máximo estacional; al no observarse gran densidad de consumidores y pérdidas de tejidos, se consideró este error poco significativo.

La uniformidad de la información obtenida permite caracterizar tres períodos en las variaciones estacionales que experimentan estas poblaciones naturales, las que se relacionan con la densidad, tamaño de las frondas y standing crop de los stands (Fig.2). 

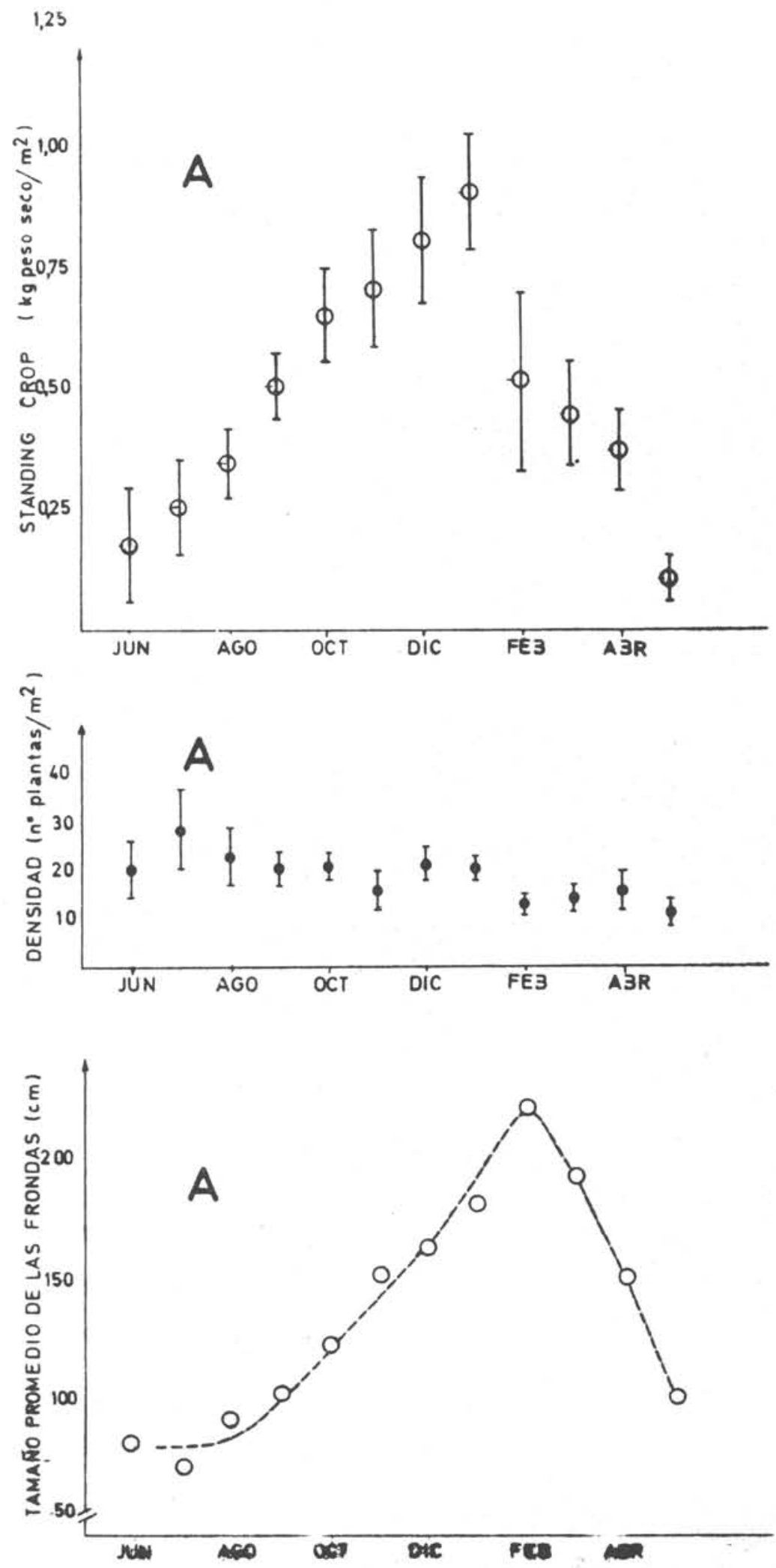

Fig. 2 - Variaciones en el tiempo de standing-crop, densıdad y tamafio promedio de las frondas en los stands estudiados. A - Stand San Vicente, centro de la población

B - Sand San Vicente, borde de la población C - Stand Bahía Coliumo. 

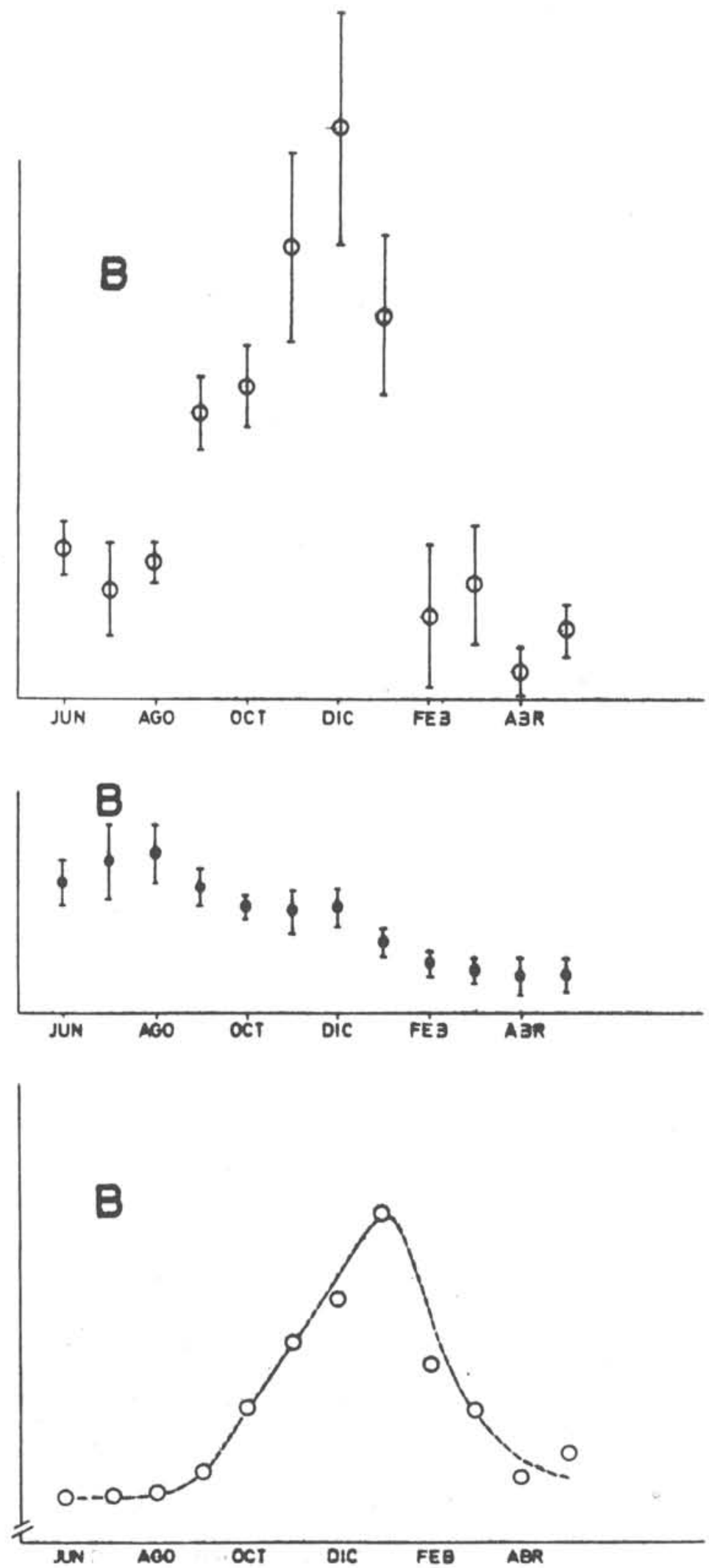

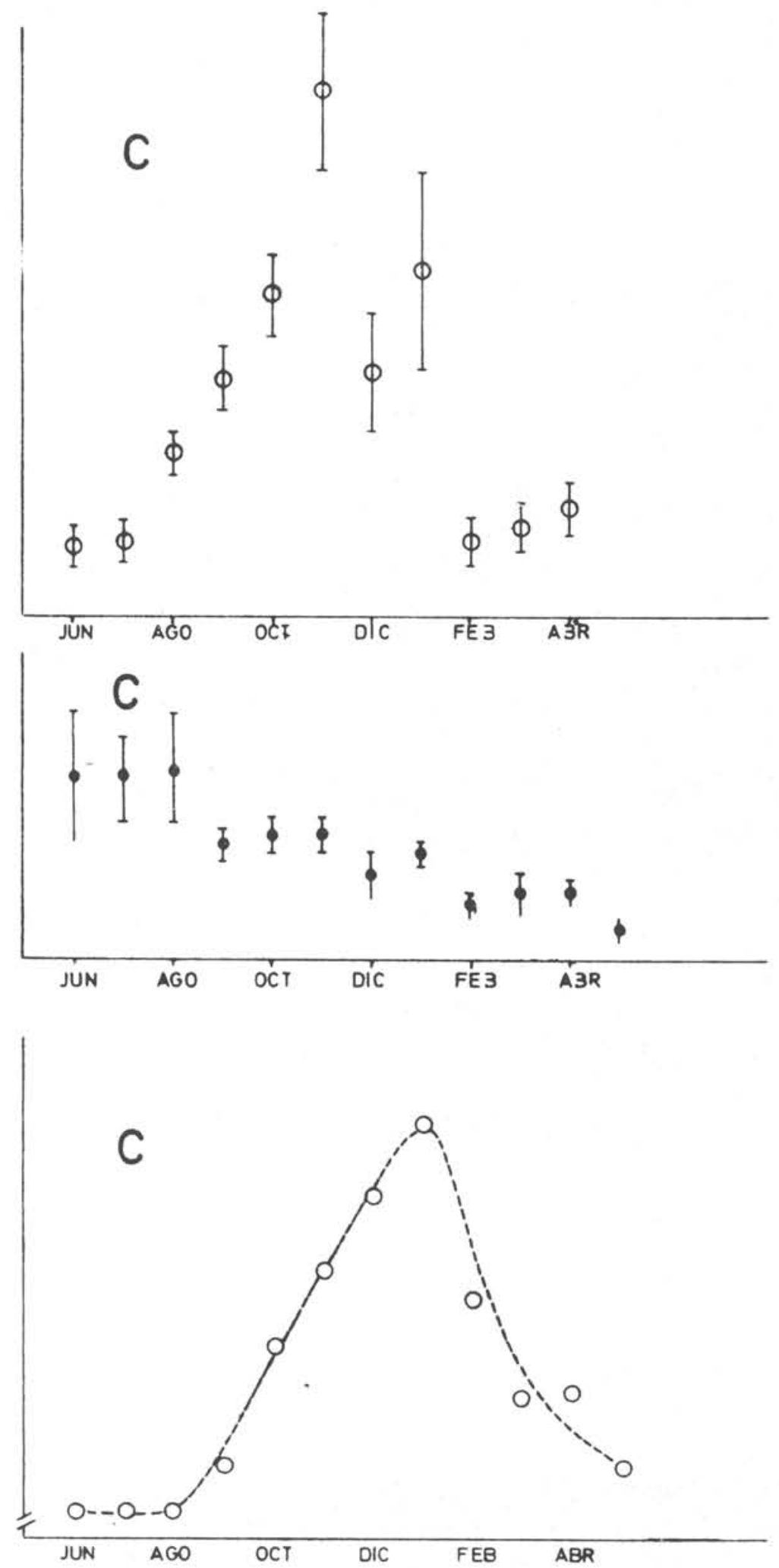

1. Periodo de Estabilidad

a) Se presenta en la estación de invierno, entre los meses de Junio, Julio y Agosto.

b) La densidad presenta valores altos, mayores a 25 plan$\operatorname{tas} / \mathrm{m}^{2}$.

c) Un standing crop que fluctúa en torno a $250 \mathrm{~g}$ peso $\mathrm{co} / \mathrm{m}^{2}$. d) El largo medio de las frondas oscila alrededor de $60 \mathrm{~cm}$.

2. Periodo de Crecimiento

a) En las estaciones de primavera y verano.

b) La estructura en el tamaño de las frondas y su largo medio experimenta un continuo incremento en este lapso.

c) Se alcanza el máximo stock estacional y anual a través 
ael aumento continuo en los valores de standing crop de $100 \mathrm{~g}$ peso $\mathrm{seco} / \mathrm{m}^{2}$ como mínimo.

d) La densidad es bastante estable y menor que en la fase anterior.

3. Periodo de Declinación

a) En los meses de fines de verano y otoño.

b) Se detecta el inicio de este periodo, al disminuir el standing crop como consecuencia de bruscas variaciones en la densidad; la presencia de una menor cobertura evita el autosombreado de los ejemplares $\mathrm{v}$ se produce un aumento en el tamaño promedio de las frondas restantes.

Los parámetros controlados experimentan fluctuaciones similares en el tiempo en las dos poblaciones estudiadas, por tal motivo, se ha generalizado el comportamiente anual de ellas (Fig. 3).

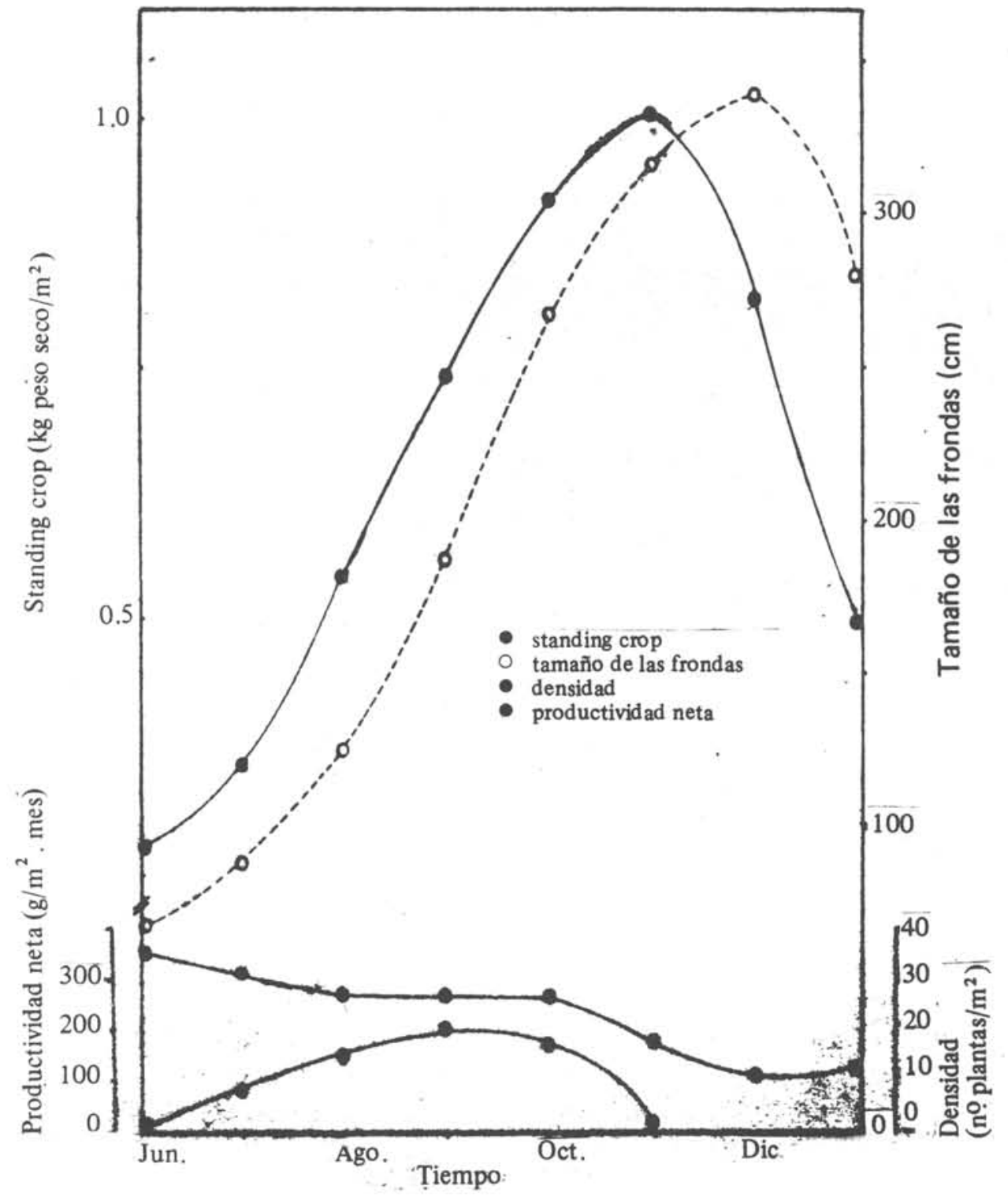

Fig. 3 - Crecimiento de Gracilaria verrucosa.

Se ha encontrado que una planta anual constituye un simil con el que podrían compararse las variaciones estacionales observadas en $G$. verrucosa.

1. Existe equivalencia en el tiempo, pues ambas tienen un crecimiento en primavera y alcanzan máximo stock estacional y anual cuando la corriente de productividaa neta tiende a cero. El mínimo estacional proporciona dos posibilidades:

a) que este sea despreciable cuando está representado por la semilla,

b) que exista una biomasa mínima en inviemo, a partir de la cual se reinicia la actividad; esta última posibilidad fue la observada en ambas localidades; no obstante, cabe suponer que en algún momento en el tiempo debe producirse el reemplazo de las fronda iejas y entonces las esporae estarían representando la situación primera.
2. Durante el período de estabilidad, la competencia entre los ejemplares se manifiesta a través de la reducción en su densidad. Las exigencias son semejantes entre los individuos de una misma especie; cualquier factor que se presente en cantidades insuficientes determina la eliminación de algunos de ellos. Todas las plantas pueden sobrevivir durante cierto tiempo en condiciones en que su crecimiento esté detenido; después alginnos de ellos son eliminados gradualmente y este raleo reduce la competencia entre las especies, casi siempre, con un mejor desarrollo y crecimiento de los eiemplares aue permanecen.

3. Las variaciones que se observan en distintas zonas de una misma población y entre poblaciones diferentes respecto al stock anual, permite caracterizarlas en forma equivalente a una planta anual por: 
a) Un maximo estacional y anual que se alcanza en menor tiempo en los márgenes de la vegetación en el centro de ella. Este máximo se alcanza con diferencias de uno a dos meses y podría estar correlacionado ya con la menor profundidad de los bordes o con la dinámica propia de un estuário, en cuanto a su aporte de nutrientes al área (Odum, 1970)

b) La presencia de dos máximos estacionales, como sucedió en Bahía Coliumo y Bahía Concepción (Fig. 2). En Bahía Coliumo, este fenómeno se puede explicar por la acción de condiciones climáticas adversas; fuertes vientos provocaron arribazones de alga al desprenderse éstas en pleno período de crecimiento. A la disminución del stock sigue am incremento al mes siguiente $\mathrm{y}$ luego deviene la declinación natural. Esta observación fue comprobada artificialmente en años posteriores, mediante la reducción mecánica del stock en la época de crecimiento y se obtuvo una prolongación de este período en el tiempo. Se homologó la situación a los continuos cortes que se realizan en un prado en que las cosechas también activan el crecimiento; cabe la posibilidad al respecto, que se altere el ritno natural de la población y como consecuencia se reduzca la productividad en el año siguiente. En Bahía Concepción, los dos máximos estacionales podrían ser consecuencia del aumento que experimenta en la misma área la población de Desmarestia sp. (Fig, 4). El stock de $G$. ve. rrucosa es afectado aquí tanto en cantidad como en calidad

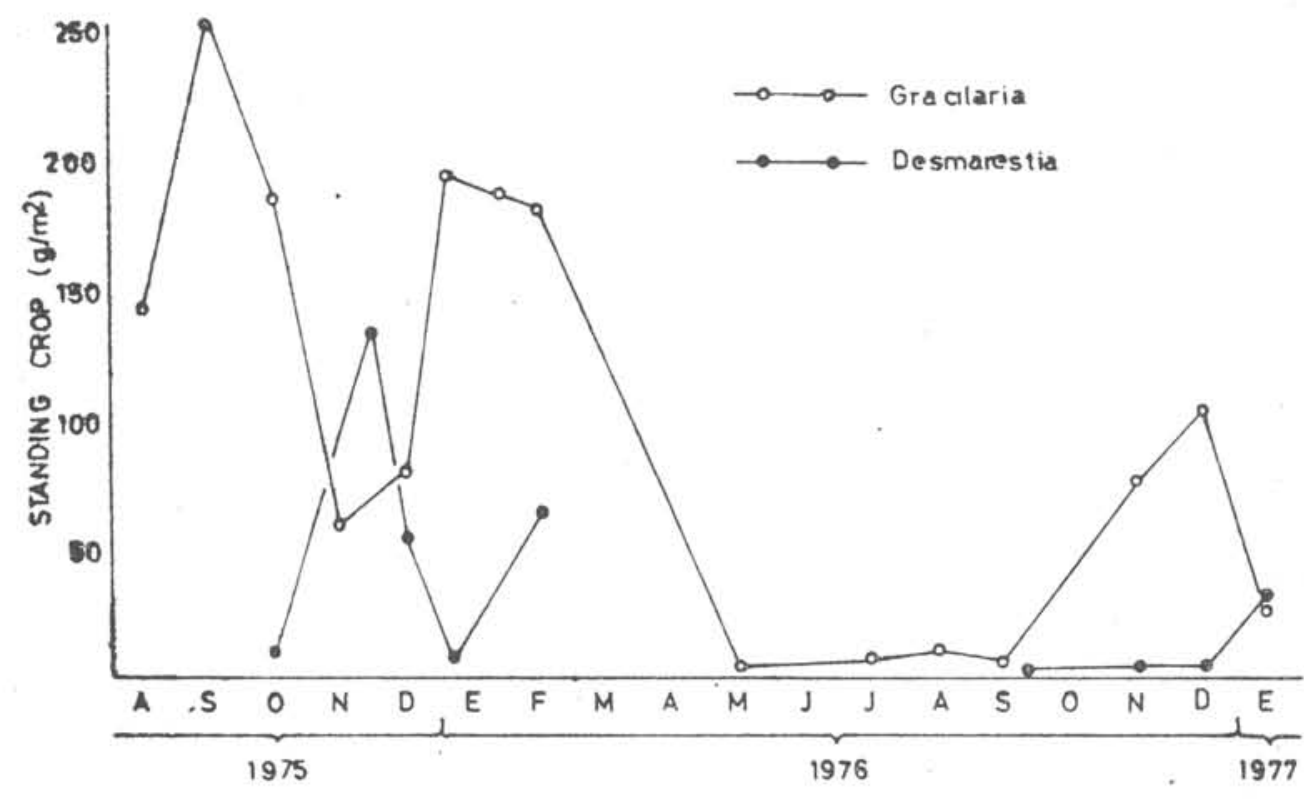

Fig. 4-Variación del standin-crop de Gracilarna verrucosa y Desmarestia sp. en Bahía Concepción.

\section{Referencias Bibliográficas}

AHUMADA, R \& CHUECAS, L, 1979. Algunas características hidrográficas de la Bahía de Concepción $\left(36^{\circ} 40 \nmid 8 ; 73^{\circ} 02^{\prime} \mathrm{W}\right)$ y áreas adyacentes. Gayana, Misc., (8): 1-56.

CAMERON, W. M. \& PRITCHARD, D. W. 1963. Estuaries. In: Hill, M. N., ed. - The sea. New York, Interscience, vol. 2, p. 306-322.

CAUSEY, N. B.; PRYTHERCH, J. P.; MCCASKILL, J.; HUMM, H. J. \& WOLF, F.A. 1944. Influence of environmental factors on Gracilaria confervoides. Bull. Duke Univ. mar. Stn, 3:19-24.

DELlAROSSA, V. 1974. Estudios ecológicos sobre Gracilaria verrucosa (Hudson) Papenfuss, 1950, en Bahía San Vicente y Bahía Dichato, Chile. Tesis de Licenciado en Biología. Chile, Universidad de Concepción, 143p.

JONES, W. E. 1959. The growth and fruiting of Gracilaria verrucosa (Hudson) Papenfuss. J. mar. biol. Ass. U. K., 38:47-56.

KATADA, M. 1963. Life forms of seaweeds and succesion of their vegetation. Bull. Jap. Soc, scient. Fish., 20:798-808.

KIM, D. H. 1970. Economically important seaweeds, in Chile. I. Gracilaria. Bot. mar., 12(2): 140-162.
- \& CANDIA, A. 1978. Una nota sobre el cultivo unialgai de Gracilaria procedente de la Isla Santa María y Río Tubul, Provincia de Arauco, Chile. Boln Soc. Biol. Concepción, 51:107-112.

ODUM, E. P. 1970. Ecologia. Mexıco, Interamericana, 639p.

OGATA, E.; MATSUI, T. \& NAKAMURA, H. 1972. The life cycle of Gracilaria verrucosa (Rhodophyceae, Gigartinales) in vitro. Phycologia, 11 (1): 75-80.

OHMI, H. 1958. The species of Gracilaria and Gracilariopsis from Japan and adjacent waters. Mem. Fac. Fish. Hokkaido Univ., 6: 1-66.

ROMO, H. \& ALVEAL, K. 1979. Estudios poblacionales en la pradera de Gracilaria verrucosa (Hudson) Papenfuss, de Isla de los Reyes, Bahía de Concepción. Cienc. Tec. Mar, Contrnes CONA, (4): 15-26.

STOKKE, K. 1957. The red algae Gracilaria verrucosa in Norway. Nytt Mag. Bot., 5: 101-111.

TSEKOS, I. \& KARATAGLIS, S. 1974. Der Einfluss der Temperatur auf das Wachstum von Karposporen-Keimlingen der Rhodophycee Gracilaria confervoides (L) Grev. Bot. mar. $17(4): 223-226$. 